\title{
Parallel Discrete Event Simulation for Multi-core Systems: Analysis and Optimization
}

\author{
Jingjing Wang, Student Member, IEEE, Deepak Jagtap, Student Member, IEEE, \\ Nael Abu-Ghazaleh, Member, IEEE, and Dmitry Ponomarev, Member, IEEE
}

\begin{abstract}
Parallel Discrete Event Simulation (PDES) can substantially improve the performance and capacity of simulation, allowing the study of larger, more detailed models, in less time. PDES is a fine-grained parallel application whose performance and scalability is limited by communication latencies. Traditionally, PDES simulation kernels use message passing; often these simulators are written for distributed environments, and shared memory is used to optimize message passing among processes on the same machine. In this paper, we develop, characterize and optimize a thread-based version of a PDES simulator on three representative multi-core platforms. The multi-threaded implementation eliminates multiple message copying and significantly minimizes synchronization delays. We study the performance of the simulator on three hardware platforms: an Intel Core i7 machine, and a 48-core AMD Opteron Magny-Cours system, and a 64-core Tilera TilePro64. We discover that the three platforms encounter substantially different bottlenecks because of their different architectures. We identify these bottlenecks and propose mechanisms to overcome them. Our results show that multithreaded implementation improves the performance over an MPI-based version by up to a factor of 3 on the Core i7, 1.4 on the AMD Magny-Cours, and 2.8 on the Tilera Tile64.
\end{abstract}

Index Terms_-PDES, Multi-threaded, Optimistic Simulation, Multi-core Systems, Optimization

\section{INTRODUCTION}

$\mathrm{D}$ ISCRETE Event Simulation (DES) is a type of simulation used to study systems where the changes of state are discrete. It is widely used in system evaluation and analysis in many areas including computer and telecommunication systems, biological networks, military war gaming, online games, and operational management. The increasing demands of simulation models challenge the capabilities of sequential simulators. Parallel Discrete Event Simulation (PDES) exploits the natural parallelism present in simulation models to substantially improve the performance and capacity of DES simulators.

The high communication overhead and latency limit the performance of PDES, especially when running on a cluster environment [1]. Several approaches have been proposed to reduce communication overheads [2], [3], [4]. However, PDES remains highly constrained by the high cost of communication.

The emergence of multi-core architectures and their expected evolution into many-cores present an opportunity for PDES and similar fine-grained applications. The low communication latency and tight memory integration among the cores on a multi-core chip substantially reduce the communication cost improving the performance and scalability of communication bound applications. However, most existing PDES simulation kernels such as WarpIV [5], GTW [6], and ROSS [7], have

- The authors are all with the Department of Computer Science, Binghamton University, Binghamton, NY, 13902.

E-mail: jwang36,djagtap1,nael,dima@cs.binghamton.edu been created for cluster environments and have not been optimized to work in multi-core settings.

In this paper, we report on our experiences in optimizing a PDES simulation kernel, the Rensselaer's Optimistic Simulation System (ROSS) [7], for multi-core platforms. Specifically, we re-implement the processbased simulator as a multi-threaded model, which we call ROSS-MT, to take advantage of the tight integration among cores on the same chip. This allows us to substantially reduce communication latency by passing events directly from one thread to another. We evaluate the performance of the multi-threaded ROSS on two primary multi-core platforms: an Intel Core i7, and an AMD Magny-Cours 48-core machine. In addition, we use a 64-tile Tilera platform as an alternative architecture.

We discover a number of performance bottlenecks, especially on the 48-core machine, and propose optimizations to reduce their effect. First, we show that the MPI barrier synchronization does not scale due to lock contention. Instead, the optimized pthread_barrier implementation should be used. Second, we show that the standard implementation of memory allocation is not aware of the non-uniform memory latency present on some multi-core architectures. We propose and evaluate several policies that are aware of these effects. Finally, we show that there is substantial contention for the incoming event queues, and present a distributed implementation that significantly reduces this contention. Together, with these optimizations, the multi-threaded ROSS outperforms the baseline distribution of ROSS by up to a factor of 3 on the Intel Core i7, and a factor of 1.4 on the 48-core AMD Magny-Cours system, and a factor of 2.8 on the Tilera platform. 
This paper significantly expands a previously published article in the International Parallel and Distributed Processing Symposium (IPDPS 2012) [8]. First, we explore the design space of the locking mechanism around the critical event queue, implementing several new organizations that differ in the locking primitives and lock distribution. In addition, we identify a significant memory leak problem that occurs due to the interaction of the NUMA optimization present in [8] with the operating system NUMA allocation policy. We develop and explore several solutions to this problem. We also present alternative NUMA optimization based on allocating space on intermediate cores. Moreover, we analyze the performance of the simulator and the proposed optimizations on a real model of a Personal Communication System. Finally, we evaluate the performance of ROSS-MT on a 64-tile Tilera platform.

The remainder of this paper is organized as follows. Section 2 provides background information on PDES in general, and the multi-core platforms used in our experiments. Section 3 provides details of the solutions proposed in this paper. Performance bottlenecks and our solutions to them are described in Section 4. Section 5 overviews the experimental setup, while Section 6 presents the performance evaluation of the ROSS-MT simulator and the proposed optimizations. In section 7 we review the related work. Finally, Section 8 offers our concluding remarks. Associated with the paper is a supplementary document that includes more background information, details regarding the experimental set up, and expanded experiments and analysis.

\section{BACKGROUND}

In this section, we first briefly describe parallel discrete event simulation and the ROSS simulator [7], and overview two multi-core platforms used in our experiments.

\subsection{Parallel Discrete Event Simulation}

In parallel discrete-event simulation (PDES), a model is partitioned across a group of Processing Elements (PEs) that communicate by exchanging time-stamped event messages [9], [10]. Each event carries a timestamp which determines the simulation time at which it is scheduled to occur. Each PE processes its events in time-stamp order to ensure causality. However, without synchronization, it is possible for an event generated from one PE to arrive at another PE after its scheduled processing time has passed; such a straggler event indicates a causality error. Two primary synchronization algorithms are widely used in PDES to enforce correct causality: conservative and optimistic synchronization. Conservative simulation requires PEs to coordinate to guarantee that no causality errors can occur. In contrast, in optimistic simulation no explicit synchronization is enforced during simulation. However, if a causality error is detected, the simulation is rolled back to a time before the straggler event, and messages are sent to cancel any erroneously sent events after that time. More details about conservative and optimistic simulation are in Section 1 of the supplementary material.

In this paper, we use the ROSS [7] PDES simulator. In the optimistic mode, ROSS leverages efficient reverse computation where, instead of check-pointing, reverse computation code is associated with every event to reverse its effect to restore the state during rollbacks.

\subsection{Multi-core Architectures}

We study three multi-core platforms, with significantly different architecture and memory organizations. The first is a 4-core Intel Core i7 processor. Each core supports two Simultaneous Multi-threaded (SMT) thread contexts. The cores have private $32 \mathrm{~KB} \mathrm{L1}$ and $256 \mathrm{~KB}$ L2 caches but share an $8 \mathrm{MB}$ L3 cache. The second platform we use is a 48-core AMD Magny-Cours machine. There are four CPU chips on the memory bus, each holding 12 cores. The chips are connected using AMD proprietary Hyper-transport 3.0 links. On each chip, the cores are located on two separate dies, with each die holding 6 cores. Each core has a private $64 \mathrm{~KB} \mathrm{L1}$ and $512 \mathrm{~KB}$ L2 caches, and shares $6 \mathrm{MB}$ L3 cache with other cores on the same die. A specialized interconnect is used to connect the caches across dies. The cores have nonuniform memory access (NUMA) to different regions in memory and experience non-uniform latencies on cache hits to the L3 cache depending on whether the cache line is in the L3 cache of the same die or a remote die. The third platform is the Tilera TilePro64, an many-core architecture with 64 identical tiled cores. Tilera features low latency and high bandwidth communication fabric interconnecting the cores. More details about the Tilera machine are presented in Section 2 of the supplementary document.

\section{Multi-Threaded ROSS: Design OVERVIEW}

In ROSS, communication occurs for three primary purposes: (1) Exchange of event messages; (2) Exchange of anti-messages, cancelling earlier messages sent erroneously; and (3) for Global Virtual Time (GVT) computation which is used to commit events, and garbage collect unneeded event checkpoint information. It is essential for communication latency to be low for all three of those functions. Otherwise, rollbacks occur more frequently, are more expensive and more difficult to contain, and GVT computation overhead becomes high, delaying event commitment and increasing the simulator memory usage.

\subsection{Communication Mechanism in the MPI-based ROSS}

MPI is used for communication in the baseline ROSS simulator (ROSS-MPI). Figure 1 shows event message 
communication mechanism in ROSS-MPI. Each PE maintains a queue of outgoing remote events. When a PE sends a message to another remote $\mathrm{PE}$, an event message is first queued into the Output Queue (Outq). Events are later dequeued from the Outq and sent to the appropriate destination process asynchronously based on receiver buffer availability. Posted sends and Posted receives buffers are used for asynchronous message passing. Once the event message is successfully received at the destination process, it is enqueued into the event queue at the receiver side. The event queue is a priority queue maintained by the scheduler to keep the events in timeorder. The scheduler dequeues events from the event queue for processing.

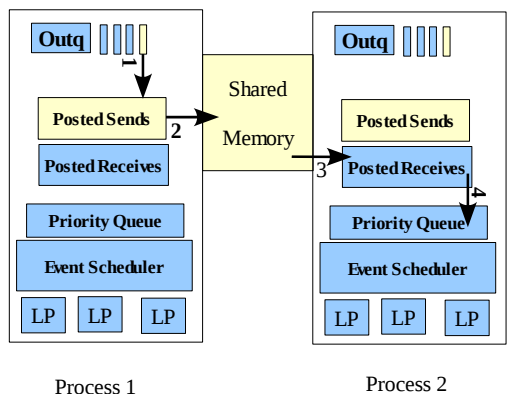

Fig. 1. MPI-based ROSS Communication Mechanism

\subsection{The Design of ROSS-MT}

In ROSS-MT, we use threads instead of processes as seen in Figure 2. Because the threads share the same address space, we use an input queue for each thread containing all remote events from other threads (PEs). No buffering is needed and thus the Posted sends and Posted receives buffers are eliminated. During communication the sender keeps each message, so that in case of rollbacks, cancellation messages can be generated. A copy of each message is then created, and a pointer to this message copy is inserted in the input event queue of the destination thread. The receiver thread dequeues events from the input queue and inserts them into the primary event queue for processing.

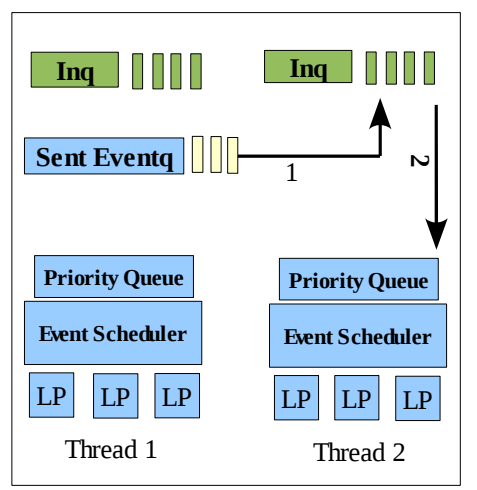

Fig. 2. Multi-threaded ROSS Communication Mechanism

\subsection{Performance of Communication Primitives}

Since PDES is a communication-bound application, the performance is substantially impacted by the performance of the communication primitives. Thus, to set the context for later results, we study the performance of the communication primitives under both message passing and multi-threaded communication for both the Intel Core i7 and the AMD 48-core platforms.

First, we perform ping-pong message exchange latency tests for both message passing and multi-threaded communication on both platforms. More precisely, the experiment consists of two MPI processes for message passing communication, or two threads for multithreaded communication, with each pinned to a different core. For the Magny-Cours platform, we selected two cores on the same die (intra-die), two cores on different dies but on the same chip (inter-die), and two cores on different chips (inter-chip) respectively. We evaluate the performance of communication primitives by measuring the message sending rate (count of exchanged messages per second). In addition, we fixed the message size at 128 bytes, which is the event message size used by ROSS.

Table 1 and Table 2 show the performance of corresponding experiments on the Intel Core i7 machine, and AMD 48-core platform respectively. We discover that the multi-threaded implementation outperforms the MPI-based version: the message rate of multi-threaded version exceeds that of MPI-based version by a factor of 3.4 on the Core i7, and 1.8 on the Magny-Cours machine. This performance improvement occurs because two memory copying operations are performed through shared memory for each message in the MPI-based communication, incurring significant overhead. On the other hand, these operations are eliminated in the multithreaded implementation.

\begin{tabular}{|c|c|c|}
\hline Communication & MPI & Multi-threaded \\
\hline inter-core & 3703704 & 12500000 \\
\hline
\end{tabular}

TABLE 1

Number of Exchanged Messages per Second on Intel Core i7 machine

\begin{tabular}{|c|c|c|}
\hline Communication & MPI & Multi-threaded \\
\hline intra-die & 980392 & 1785714 \\
inter-die & 833333 & 1492537 \\
inter-chip & 806452 & 1470588 \\
\hline
\end{tabular}

TABLE 2

Number of Exchanged Messages per Second on AMD 48-core machine

\section{Performance Bottlenecks and Opti- MIZATIONS}

In the next set of experiments, we use the Phold benchmark [11] to compare the performance of the baseline 


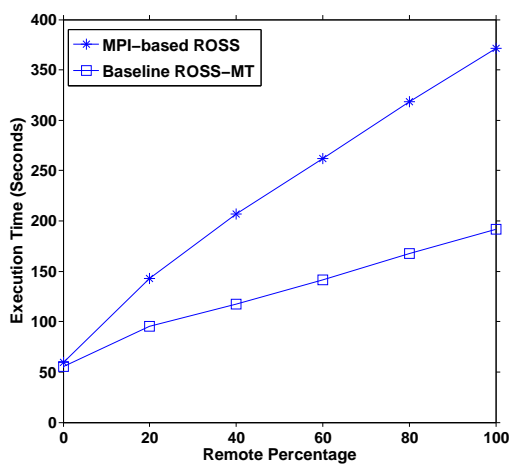

(a) Core i7

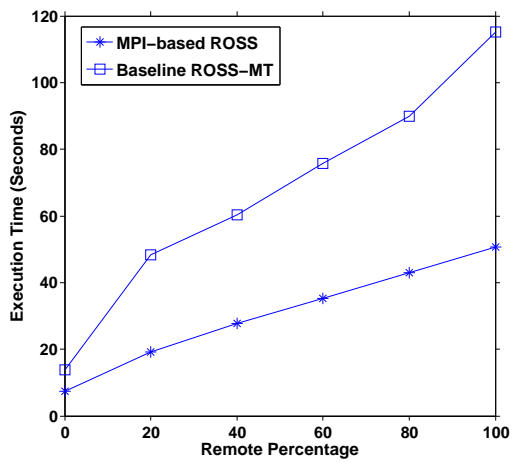

(b) Magny-Cours

Fig. 3. Performance of Baseline ROSS-MT vs. ROSS using MPI

multi-threaded implementation to the MPI implementation, as shown in Figure 3. Phold is the most widely used benchmark for performance evaluation of PDES systems [12], [13]. The model starts with a number of objects that have events. Event execution sends a message to another object (picked uniformly among all the objects in the simulation). The message causes this object in turn to later send another event message to a third object. Thus, the number of events in the simulation remains constant. Phold can be configured to control the percentage of event messages that are remote. More details about Phold and the experimental setup are presented in Section 5. While the Core i7 results show substantial performance improvements with multithreading, surprisingly, the Magny-Cours results show considerable slowdown. Thus, the section identifies the performance bottlenecks in the multi-threaded implementation, and provides optimizations to address them.

\subsection{Efficient Barrier Synchronization}

Barrier synchronization and all-reduce communication primitives are key components of the GVT computation. ROSS-MT uses its own library for barrier synchronization and all-reduce operation. In the baseline version of the multi-threaded implementation, we used condition variables and pthread_mutex for implementing these operations. We found that the use of condition variables can result in high overheads. We addressed this problem by using the pthread_barrier implementation instead of condition variables.

\subsection{NUMA-aware Free Memory Management}

ROSS implements application-level free memory management to avoid unnecessary use of the memory allocation library. The ROSS implementation places the memory of an event message after it is consumed in a free memory pool. This memory is then used for future message events. Suppose that a message is generated from PE 1 to PE 2. The message is allocated by PE 1 from its closest memory region (the Operating System NUMA option enforces that). Once the message is consumed by PE 2, it is returned to the memory pool for PE 2. In the future, if PE 2 needs to send an event to another PE, say PE 3, it picks the memory region that was allocated by PE 1, which is remote for both PE 2 and PE 3, leading to high access latencies.

To address this problem, we propose splitting the free memory pool to keep track of the allocation source. When PE 2 needs memory space for an event, it uses the free memory pool for the destination PE to ensure NUMA friendly behavior. If there is no available memory for the destination PE, the memory region is picked from the free queue of the sender PE itself. As a result, every event message is local to either the source or the destination. In addition, we implemented a Last In First Out (LIFO) approach to message allocation to improve cache reuse. We call this allocator the Base NUMA allocator (BNA).

While BNA is effective, it suffers from the following problem. If the communication activity is not balanced between any given pair of PEs, a subtle memory leak occurs. Consider a chain topology with 3 PEs such that PE 1 communicates to PE 2, PE 2 communicates to PE 3 , and PE 3 communicates back to PE 1 . PE 1 receives

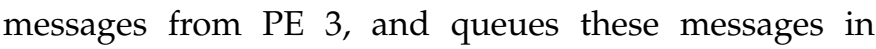
the free memory queue for PE 3. However, it never communicate to PE 3, so these messages just accumulate in this queue. PE 1 never receives messages from PE 2 , so it has no free message in the memory pool for PE 2 to use, and is forced to allocate memory from its own free-memory pool. Eventually, this memory-pool is consumed, and no memory is available to communicate to PE 2. The same pattern repeats at PE 2 and PE 3 . The simulation eventually enters livelock as it computes GVT and reclaims checkpoint memory, discovering that none is available. Thus, the NUMA optimization combined with the OS allocation policy leads to catastrophic failure of the simulation.

To address this problem, we monitor the size of each memory pool during simulation. If the memory pool grows beyond a threshold, we return this memory to the originating PE's free-memory pool (free queue). This operation requires simple pointer manipulation to connect the free-memory linked-list to the originating PE's freememory list. We call this BNA policy Free Queue (BNA$F Q$ ). If the value of the threshold used for memory return 
is too small, we return memory frequently, incurring overheads. On the other hand, if the threshold is large, the memory leak may still occur. Empirically, we find that there is a relatively wide range of this threshold that performs well on both platforms. In our experiments, we used a threshold of 50000 events in the memory pool.

BNA-FQ has the following disadvantage: each PE must acquire a lock when it accesses its own free memory pool, introducing significant overhead. To reduce the lock overhead, we use an intermediate staging buffer where the events are moved to first. When a local PE memory pool is exhausted, the PE picks up the events placed for it in this buffer. Thus, contention (and locking) occurs only when accessing the staging buffer, which is an infrequently occurring operation. At the same time, the frequently accessed local free-memory pool remains private, and requires no locking. We call this BNA allocator Staging Buffer (BNA-SB).

Finally, we implemented another NUMA-aware policy that allocates event memory from a memory bank between the sender and the receiver to reduce and balance the NUMA overhead; we call this allocator Delay Sensitive NUMA (DSN). We implemented DSN on the 48-core platform which has 8 memory banks. Each PE has a preference list for each destination NUMA node. The list keeps the selection preference for the memory bank to allocate events. The list is created based on the NUMA node distance matrix on the 48-core machine. The first memory bank in the list has the minimum total distance between itself and, both sender and receiver nodes. The other banks in the list are sorted accordingly. The lookup of the memory bank starts from the front of the list. If the selected memory bank is completely in use, the next memory bank in the list is selected. In order to prevent memory leaks, the memory bank for the sender node is selected only if the memory banks for other nodes are empty.

\subsection{Distributed Locking for the Input Queue}

By allowing the sender threads to directly access the input queue of the receiving threads, we eliminate the need for a buffer copy to an intermediate message queue. However, each input queue may now be accessed by any of the sender threads, as well as the receiving thread (i.e., all threads in the simulation). This gives rise to high contention on the lock to access the input queue. To reduce this contention, we split the input queue into private queues, one for each possible sender. The contention for the queue is reduced from all threads, to only two threads, the sender and the receiver. To reduce the locking overhead, we used the pthread spin lock.

\subsection{Lock Implementation Tradeoffs}

To reduce the locking overhead, we attempted to use reader-writer locks in the fully distributed case. The sender threads are readers (they can all access the distributed queue at the same time since none of them accesses the same queue) and the receiver thread is a writer (it gets exclusive access to the queues). In this way, only one lock needs to be acquired by the receiver. In this design, there is no contention between the different sender threads since each goes to a different queue. However, there is a contention between the sender threads and the receiver thread since they may be accessing the same queue at the same time. To combine the advantages of both pure distributed locking and reader-writer locking, we also developed a hybrid locking approach which requires a few locks, and introduces small lock contention. In this design, the receiver has 8 reader-writer locks, each competed by 6 sender threads on the 48-core platform.

\section{Experimental Setup and Benchmark}

We use the Phold benchmark [11], for most of the experiments. Before the execution of the simulation, the freememory pool is set to be of size 150000 events at each PE; this pool is reused during the simulation. In addition to remote percentage, the number of initial events per object (we selected 1 for this parameter) can also impact the performance as it increases the number of events in the simulation.

Some of our experiments also use a Personal Communication System (PCS) model [14] in order to demonstrate that the trends hold when considering real simulation models. The PCS model simulates realistic handoff patterns in PCS networks. In this model, an event simulates a mobile phone call being generated at a cell phone tower, and later sent to another tower. A new phone call may be generated at the end of the previous one. At the end of the simulation, the model collects performance statistics such as how often calls were blocked because of limited tower capacity.

We used both Intel Core i7 and 48-core AMD MagnyCours as our primary platforms, to evaluate performance of multi-threaded ROSS against MPI based ROSS (ROSSMPI). In addition, some experiments were performed on a 64-tile Tilera TilePro64 platform (see Section 4 of the supplementary document). More details about the configurations on each multi-core are in Section 2 of the supplementary document. In the next section, we evaluate the Phold performance on these three different architectures.

\section{Performance Evaluation}

In this section, we present an experimental evaluation of ROSS-MT and the different optimizations we proposed. Before we present these results, we first evaluate the performance of ROSS-MT with different lock strategies and different NUMA memory management policies, to identify the most efficient implementations. Once we identify these implementations, we use them in the remainder of the experiments. For all results, each point represents an average of 10 separate runs, which we verified was sufficient to bound the $95 \%$ confidence interval to be within $2 \%$ of the average. 


\subsection{Lock overhead of ROSS-MT}

Figure 4(a) shows the execution time of ROSS-MT when using the distributed locking on the Magny-Cours platform. Figure 4(b) shows the corresponding simulation efficiency. Efficiency is calculated as the ratio of committed events to the total events executed. With a single queue, high lock contention occurs, leading to starvation and low efficiency of the simulation. We observed that a thread fails to acquire a lock for a long period of time, causing substantial delays until it manages to send its event, causing a long rollback once this late event is received. As we increase the number of queues, the lock contention is reduced and the efficiency increases. Increasing the queues further eventually results in slightly lower performance, as no significant contention is encountered, while the receiver is burdened with having to acquire a higher number of locks to check all the different queues.

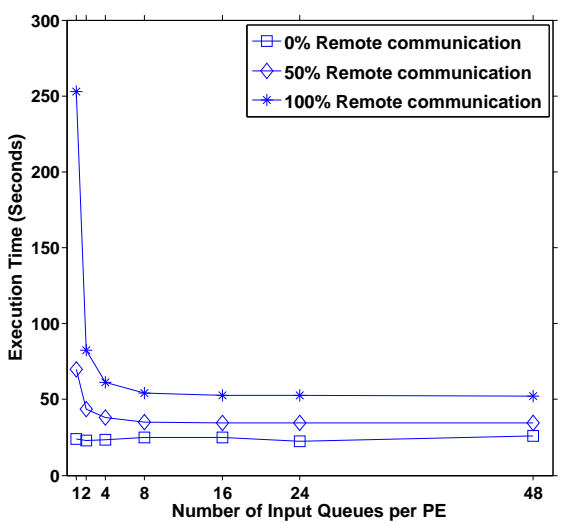

(a) Execution Time

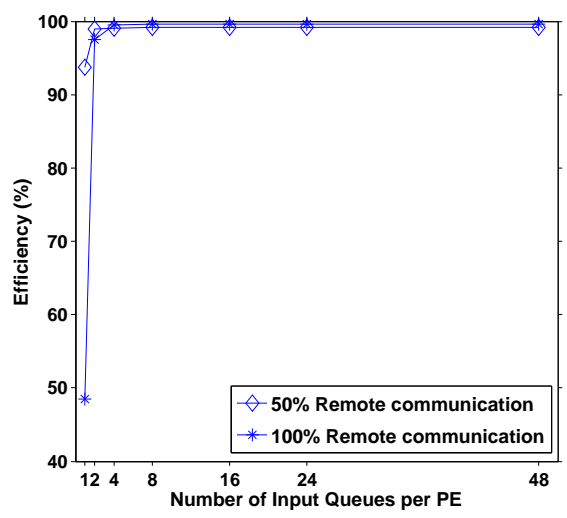

(b) Efficiency

Fig. 4. Impact of Distributed Locking: Magny-Cours

Figure 5 shows the performance of ROSS-MT with different locking strategies under different remote communication percentages. It is clear that ROSS-MT with spin-lock performs better than both the mutex and the reader-writer lock. As the remote percentage increases, the version with reader-writer lock performs worse than the other two variations, because the writers experience starvation.

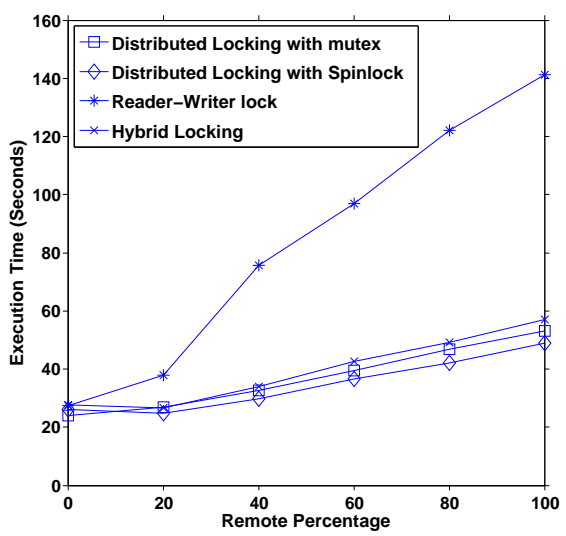

Fig. 5. Impact of Locking Organization: Magny-Cours

\subsection{Evaluation of NUMA Policies}

The default memory policy on the 48-core NUMA machine is first-touch, where a page of data is allocated in the memory of the core that first accesses it [15]. This policy may cause a page to be returned to the free pool used by a different node than the node it is pinned to. A better solution for memory allocation is to ensure that the pages are allocated on a specific NUMA node by using the NUMA library APIs. Figure 6 shows the performance of ROSS-MT with different memory allocation policies. The specific NUMA node policy achieves up to $10 \%$ performance improvement compared to first-touch policy. We use this NUMA-aware memory allocation policy in our later experiments.

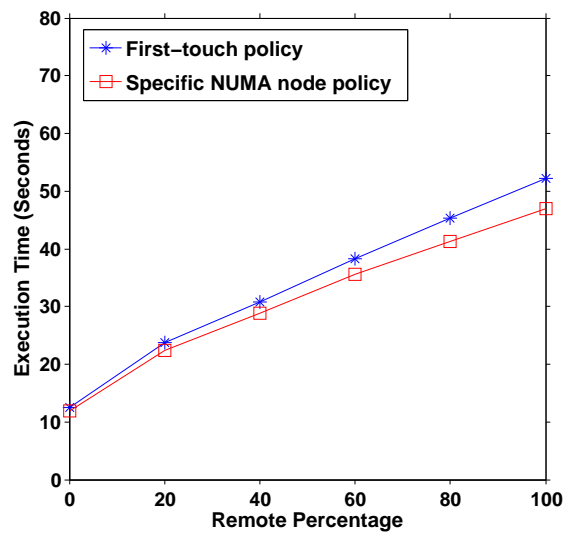

Fig. 6. Impact of Memory Allocation policies: MagnyCours

Figure 7 shows the performance of 48-way simulation under different NUMA policies on the Magny-Cours machine. In this experiment, we compare four different implementations of ROSS-MT: the version without NUMA optimization, BNA-FQ, BNA-SB, and DSN. Recall that both BNA-FQ and BNA-SB employ the same BNA allocation described in Section 4, but use different approaches to return memory in the case of asymmetric communication (solving the memory leak problem we identified in Section 4). In particular, when a PE's memory pool grows beyond a threshold, BNA-FQ allows 
the $\mathrm{PE}$ to return the memory to the originating $\mathrm{PE}^{\prime} \mathrm{s}$ free memory pool. On the other hand, BNA-SB returns the memory of freed events to an intermediate staging buffer. A PE later picks up the events placed for it in the buffer when its memory pool is exhausted. Finally, DSN uses a different NUMA allocation policy where each PE maintains a preference list for each destination NUMA node.

Figure 7 shows that BNA-FQ performs poorly in comparison to both BNA-SB and DSN, and even worse than the implementation with no NUMA optimization. After analysis, we discovered that BNA-FQ suffers high overheads because of lock contention during the access to the free-memory pools. This contention is dramatically reduced by the staging buffer used in BNA-SB. BNA-SB performs marginally better than DSN (which allocates memory halfway between the sender and receiver); the NUMA latency between caches in our 48-core platform is not that different, providing little opportunity for DSN to improve performance. We believe DSN may become more beneficial on other architectures with more heterogeneous latencies at the cache level. We use BNA$\mathrm{SB}$ for ROSS-MT in the remaining experiments.

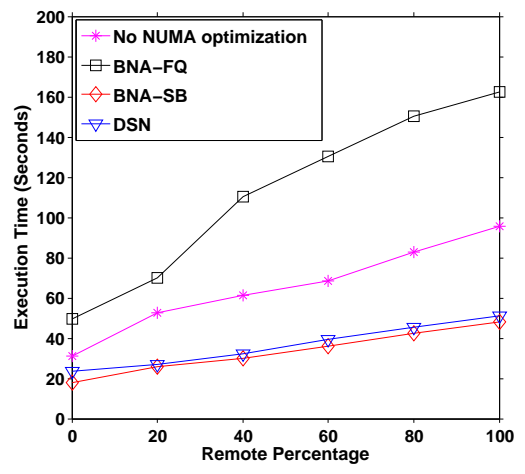

Fig. 7. Impact of NUMA policies: Magny-Cours

\subsection{Impact of the Optimizations}

We implemented the optimizations discussed in the previous section (Efficient barrier synchronization, NUMA aware memory pool management, distributed input queue, and lock optimization). Figure 8(a), Figure 8(b) and Figure 8(c) show the performance improvement obtained from each of the optimizations in isolation and combined on the Core i7. We consider a 2-way, 4-way and 8-way simulation, while keeping the number of objects per thread the same. A number of interesting observations can be made. For two nodes, as the number of remote messages increases, the optimizations harm performance. Distributing the locking on the queue is not beneficial since the degree of contention is not significant, but the overhead is increased. Moreover, NUMA issues are not important either since each memory element is local to either of the two threads. Finally, lock contention issues are minor in the barrier implementation. It is interesting to see some gain initially, but that is likely due to the LIFO strategy introduced as part of the NUMA optimization; other optimizations introduce overhead without benefit for a two-thread simulation. As the number of threads is increased, the optimizations start to become useful. The optimized ROSS-MT achieves up to $50 \%$ improvement relative to the baseline ROSSMT.

Figure 9(a), Figure 9(b) and Figure 9(c) show the impact of the optimizations for 4, 16 and 48 thread scenarios respectively on the Magny-Cours. Since the bottlenecks were most severe for this machine, the optimizations yield substantial improvement in performance (over $150 \%$ for 48 threads). The impact of the barrier optimization increases with the degree of parallelism, and reduces slightly with the increase in event communication (recall that the barrier optimization affects GVT computation but not event communication). We also study the impact of the optimizations on the MagnyCours when the percentage of remote communication is fixed. Because of space limitations, we present these results in Section 3 of the supplementary material.

In the next experiment, we evaluate the performance of ROSS-MPI and the optimized ROSS-MT on three platforms, using the classical Phold model, as shown in Figure 10. In Figure 10(a), we show results on the Core i7 machine. In particular, the experiments were executed on 8 hardware threads. It is clear that the multithreaded implementation is substantially faster than the MPI version on this platform. Figure 10(b) shows the same comparison for the Magny-Cours platform with 48 cores. ROSS-MT also outperforms the MPI version, although the gap is substantially smaller. Figure 10(c) shows the performance of ROSS-MPI and ROSS-MT on the Tilera machine. The simulation model consists of 56000 objects equally distributed across 56 PEs. In addition, the simulation time was set to 1000 for Tilera. Clearly, the performance of ROSS-MT exceeds that of ROSS-MPI by a factor of up to 2.8 on the Tilera platform. More detailed evaluation of the simulator on the Tilera machine is presented in Section 4 of the supplementary material.

\subsection{Other Benchmarks}

In our previous experiments, we used the Phold benchmark to evaluate the performance of both ROSS-MPI and ROSS-MT. In this study, we consider another two benchmarks: Personal Communication Services (PCS) simulation model [14], and hierarchical Phold model [16]. Both of these benchmarks mimic the behaviors of realworld systems.

In the PCS model, the total number of cell phone towers (LPs) is fixed at 57600. Figure 11(a) and Figure 11(b) show the PCS model performance on the Core i7 and Magny-Cours machines respectively. The sequential simulation run-time was 1106 seconds on the Core i7 machine, and was 247 seconds on the Magny-Cours machine. We discover that the performance improvement of ROSS-MT over ROSS-MPI in the PCS model is 


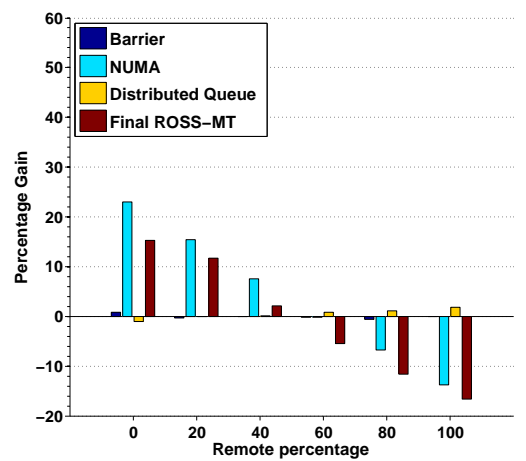

(a) 2 Nodes

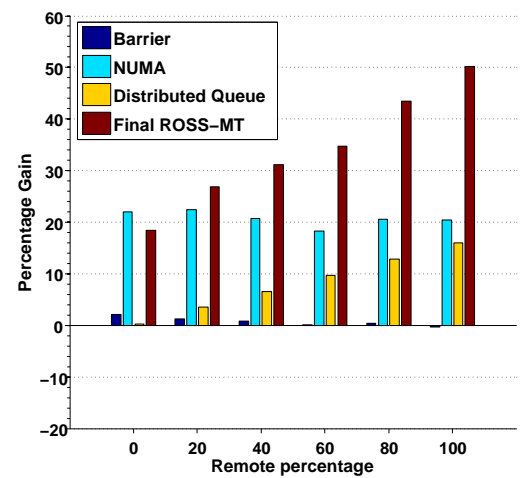

(b) 4 Nodes

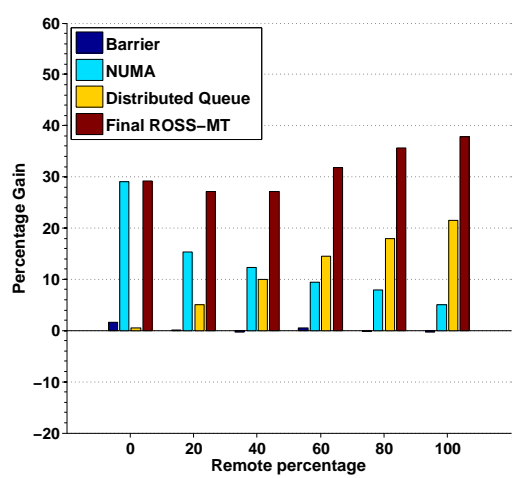

(c) 8 Nodes

Fig. 8. ROSS-MT on the Intel Core i7

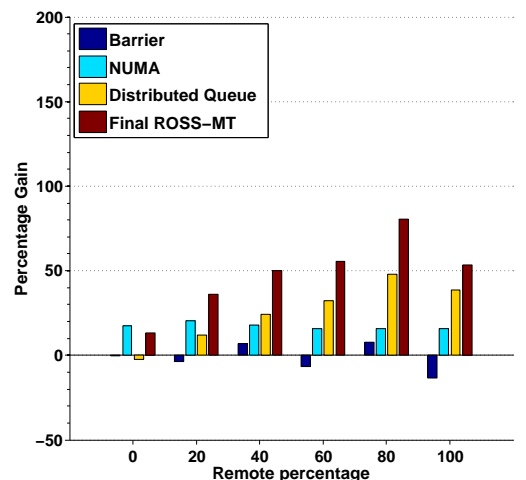

(a) 4 Nodes

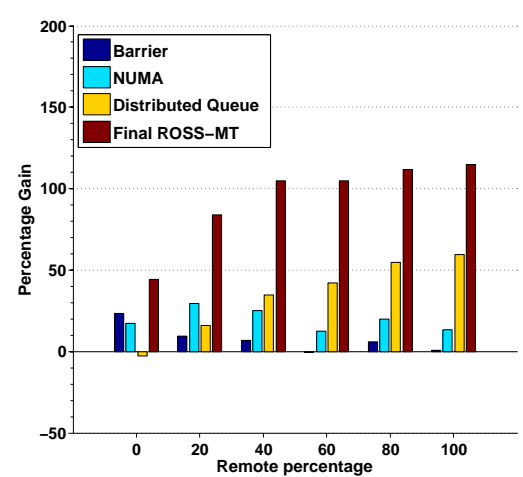

(b) 16 Nodes

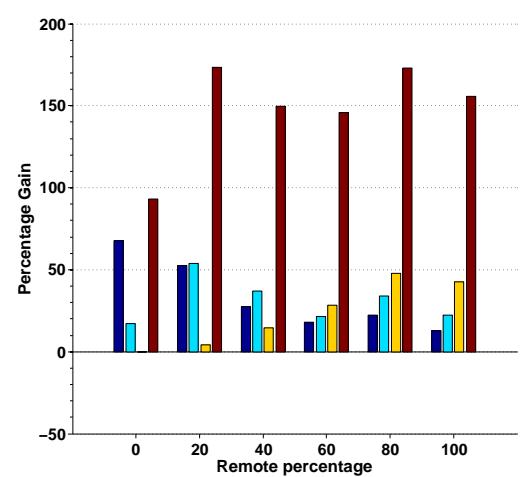

(c) 48 Nodes

Fig. 9. Magny-Cours performance with increased parallelism

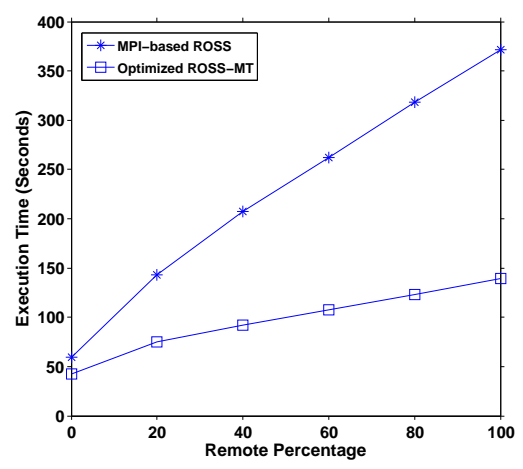

(a) Intel Core i7

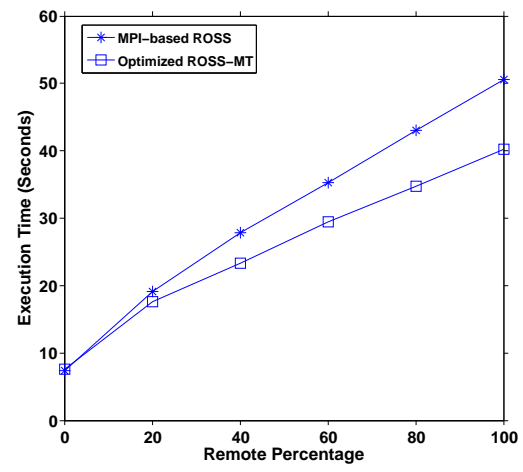

(b) AMD Magny-Cours

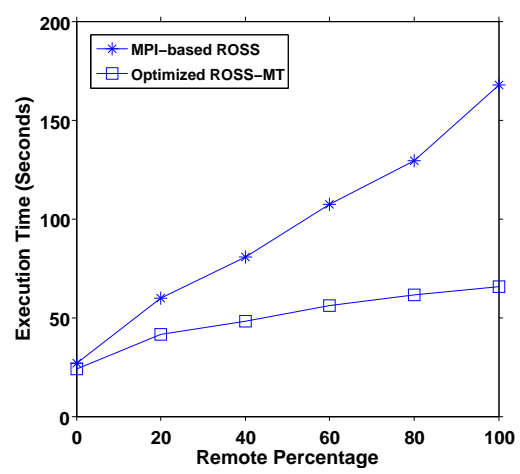

(c) Tilera

Fig. 10. Optimized ROSS-MT vs. ROSS-MPI

smaller than that in the Phold model. This is because that PCS model has more computation and less remote communication $(<10 \%)$ than Phold.

Hierarchical Phold model groups objects in a hierarchical communication structure, compared to the random selection of communication targets in the classical Phold. The communication frequencies among groups of objects are determined by a Pareto distribution [16]. This model exhibits similar object communication graphs to those present in some real-world systems such as 3M-ncs, an Internet-scale network simulation model [17]. In our experiment, the hierarchical Phold model consists of 8160
LPs distributed equally among PEs. Figure 12 shows the performance of hierarchical Phold model for both ROSSMPI and ROSS-MT on the 48-core platform. Clearly, ROSS-MT achieves a better performance than ROSS-MPI. For example, at the case of 48 nodes, the performance of ROSS-MT exceeds that of ROSS-MPI by a factor of 1.4 .

\section{Related Work}

PDES is difficult to parallelize because of its fine-grained nature, and dynamic dependency patterns which vary with the model being simulated [9], making it substan- 


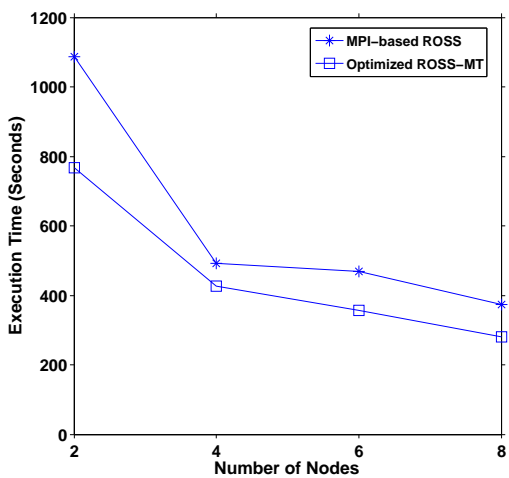

(a) Core i7

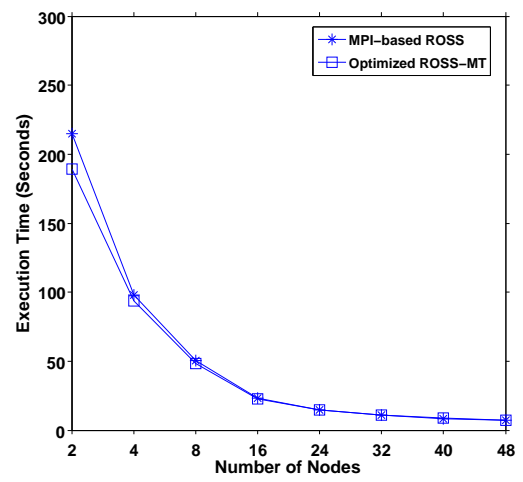

(b) Magny-Cours

Fig. 11. The Performance of PCS Model

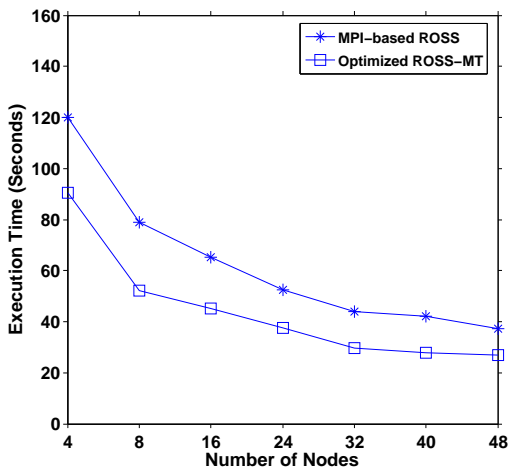

Fig. 12. Hierarchical Phold Model Performance on the AMD Magny-Cours

tially different from typical parallel applications. In this study, we focus on optimizations specific to PDES.

\subsection{Optimizing the Communication Cost for PDES}

Bahulkar et al. [16] proposed a static partitioning approach to reduce the communication overhead of PDES, by placing the most heavily communicating objects on the same processor before the simulation starts. Similarly, dynamic partitioning have been proposed to repartition the simulation to recover dynamic behavior changes of the simulation model for both conservative (e.g., [18]) and optimistic (e.g., [3]) synchronization protocols. Chetlur et al. [4] proposed the use of message aggregation, where multiple event messages are combined in a single communication message, to amortize the overheads associated with communication across multiple messages. Mattern developed a non-blocking GVT algorithm which allows event processing to proceed concurrently with GVT computation, allowing the cost of that expensive operation, which includes global communication among the PEs, to be hidden [19]. Noronha et al. used a programmable network card to optimize event communication and GVT computation [20].

\subsection{MPI on Shared Memory Architectures}

This work replaces MPI communication with efficient shared memory primitives. Thus, it is important to understand the advantages of this model over optimizing MPI operation for shared memory. Typically, MPI implementations require two memory copy operations to copy the message from the sender to the shared memory segment and then back to the receiver. Flow control is supported using pipes to ensure that the message is received correctly. In addition, implementing the semantics of message passing requires MPI to use multiple system calls for each send and receive. The message is piped (using a system call) through the shared memory segment to avoid a case where the message size exceeds the available space.

Some researchers have proposed different approaches to improve the performance of MPI for shared memory architectures. Graham et al. [21] optimized collective operations of MPI, where each process can directly access other processes data out of their shared memory buffers. Hoefler et al. [22] leveraged remote memory access interface of MPI-3.0 to support the direct memory access for one-sided communication. However, double copies are required for point-to-point communication. Goglin et al. [23] support efficient intra-node MPI communication for large messages, by using kernel-assisted direct copies between processes. However, for small messages (such as those used in PDES), they observe that the standard two-copy implementation performs better.

\subsection{Multi-threaded PDES}

To improve PDES performance on multi-cores, some researchers have designed multi-threaded PDES engines. Chen et al. [24] proposed a multi-threaded PDES implementation that uses a global event scheduling mechanism. Although such an organization is helpful for load balancing, it sacrifices locality and introduces overheads for scheduling through a centralized queue. Vitali et al. [25] proposed a different multi-threaded PDES simulator that employed a load-sharing scheme. This simulator differs from ROSS-MT because multiple threads may be assigned to the same PE. An interesting direction of future work is to comparatively evaluate these different organizations of the multi-threaded simulation engine. Wang et al study the performance of PDES on clusters of multi-cores, discovering that inter-machine latency dominates, but that solutions can be developed to hide its very high cost [26]. 


\section{Conclusions and Future Work}

This paper presented experiences in building a multithreaded PDES simulator optimized for representative state-of-the-art multi-core machines. We used the ROSS PDES simulator, and modified it from a process-based model to a thread-based model. Although the implementation showed significant performance benefits on the Core i7 platform, it showed surprisingly poor performance on the AMD Magny-Cours.

We studied the reasons for this poor performance, and identified three bottlenecks. First, the barrier and allreduce primitives used in GVT computation were implemented in an inefficient way using condition variables and broadcasts. We replaced this implementation with one that uses the pthread_barrier mechanism, which uses atomic instructions for efficiency. The second performance problem occurred due to the NUMA nature of the Magny-Cours platform. We proposed and evaluated a number of policies that are sensitive to the NUMA nature of the platform. The best solution results in guaranteed liveness, and incurs no performance overhead when it is not needed. The third bottleneck was due to the lock contention on the input queue. We resolved this issue by splitting the queues to reduce contention. We also studied the tradeoff between different lock implementations, exploring the use of mutex locks, spinlocks and reader-writer locks. The optimizations resulted in substantial improvement in performance; optimized ROSS-MT outperforms the MPI-based version by a factor of up to 3 on the Core i7 platform, and up to 1.4 on the Magny-Cours, and up to 2.8 on the Tilera.

In our future work, we plan to explore some lock-free alternatives to reduce coordination overheads. Moreover, we plan to explore some adaptive mechanisms to automatically enable and configure the different optimizations to match the architecture and model behavior.

\section{ACKNOWLEDGEMENTS}

This material is based on research sponsored by Air Force Research Laboratory under agreement number FA8750-11-2-0004. We also gratefully acknowledge support from the National Science Foundation grants CNS0916323 and CNS-0958501.

\section{REFERENCES}

[1] J. Wang, D. Ponomarev, and N. Abu-Ghazaleh, "Performance analysis of multithreaded PDES on a cluster of multicores," in Proc. ACM/IEEE/SCS International Workshop on Principles of Advanced and Distributed Simulation (PADS), 2012, (short paper).

[2] L. Li and C. Tropper, "A design-driven partitioning algorithm for distributed Verilog simulation," in Proc. 20th International Workshop on Principles of Advanced and Distributed Simulation (PADS), 2007, pp. 211-218.

[3] P. Peschlow, T. Honecker, and P. Martini, "A flexible dynamic partitioning algorithm for optimistic distributed simulation," in Proc. 20th International Workshop on Principles of Advanced and Distributed Simulation (PADS), 2007.

[4] M. Chetlur, N. Abu-Ghazaleh, R. Radhakrishnan, and P. A. Wilsey, "Optimizing communication in Time-Warp simulators," in 12th Workshop on Parallel and Distributed Simulation. Society for Computer Simulation, May 1998, pp. 64-71.
[5] WarpIV Technologies (J. Steinman et al), "The warpiv parallel simulation kernel version 1.5.2," 2008, software available from http://www.warpiv.com/.

[6] S. Das, R. Fujimoto, K. Panesar, D. Allison, and M. Hybinette, "GTW: a Time Warp system for shared memory multiprocessors," in Proceedings of the 1994 Winter Simulation Conference, J. D. Tew, S. Manivannan, D. A. Sadowski, and A. F. Seila, Eds., Dec. 1994 pp. 1332-1339.

[7] C. Carothers, D. Bauer, and S. Pearce, "ROSS: A highperformance, low memory, modular time warp system," in Proc of the 11th Workshop on Parallel and Distributed Simulation (PADS), 2000.

[8] D. Jagtap, N.Abu-Ghazaleh, and D.Ponomarev, "Optimization of parallel discrete event simulator for multi-core systems," in Proc. of IPDPS, 2012.

[9] R. Fujimoto, "Parallel discrete event simulation," Communications of the ACM, vol. 33, no. 10, pp. 30-53, Oct. 1990.

[10] A. J. Park and R. M. Fujimoto, "Efficient master/worker parallel discrete event simulation on metacomputing systems," IEEE Transactions on Parallel and Distributed Systems, vol. 23, pp. 873880, 2012.

[11] R. Fujimoto, "Performance of time warp under synthetic workloads," Proceedings of the SCS Multiconference on Distributed Simulation, vol. 22, no. 1, pp. 23-28, Jan. 1990.

[12] K. Perumalla, "Scaling time warp-based discrete event execution to $10^{4}$ processors on a blue gene supercomputer," in Proc. of the ACM Conference on Computing Frontiers (CF), 2007.

[13] D. Bauer, C. Carothers, and A. Holder, "Scalable time warp on bluegene supercomputer," in Proc. of the ACM/IEEE/SCS Workshop on Principles of Advanced and Distributed Simulation (PADS), 2009.

[14] C. Carothers, R. Fujimoto, and Y. Lin, "A case study in simulating pcs networks using time warp," in Proc. Workshop on Parallel and Distributed Simulation (PADS), Jun. 1995, pp. 87-94.

[15] C. McCurdy and J. Vetter, "Memphis: Finding and fixing numarelated performance problems on multi-core platforms," in Performance Analysis of Systems \& Software (ISPASS), 2010 IEEE International Symposium, March 2010, pp. 87 - 96.

[16] K. Bahulkar, J. Wang, N. Abu-Ghazaleh, and D. Ponomarev, "Partitioning on dynamic behavior for parallel discrete event simulation," in Proc. ACM/IEEE/SCS International Workshop on Principles of Advanced and Distributed Simulation (PADS), 2012.

[17] B. Hou, Y. Yao, and S. Peng, "Empirical study on entity interaction graph of large-scale parallel simulations," in Proc. of the ACM/IEEE/SCS Workshop on Principles of Advanced and Distributed Simulation (PADS), 2011.

[18] A. Boukerche and S. Das, "Dynamic load balancing strategies for conservative parallel simulation," in Proc. 11th Workshop on Parallel and Distributed Simulation (PADS), 1997, pp. 32-37.

[19] F. Mattern, "Efficient algorithms for distributed snapshots and global virtual time approximation," Journal of Parallel and Distributed Computing, vol. 18, no. 4, pp. 423-434, Aug. 1993.

[20] R. Noronha and N. B. Abu-Ghazaleh, "Active NIC optimization for Time Warp," in International Parallel and Distributed Processing Symposium (IPDPS), 2002.

[21] R. L. Graham and G. Shipman, "Mpi support for multi-core architectures: Optimized shared memory collectives," in Proceedings of the 15th European PVM/MPI Users' Group Meeting on Recent Advances in Parallel Virtual Machine and Message Passing Interface, 2008, pp. 130-140.

[22] T. Hoefler, J. Dinan, D. Buntinas, P. Balaji, B. Barrett, R. Brightwell, W. Gropp, V. Kale, and R. Thakur, "Leveraging mpi's one-sided communication interface for shared-memory programming," in Proceedings of the 19th European conference on Recent Advances in the Message Passing Interface, 2012, pp. 132-141.

[23] B. Goglin and S. Moreaud, "Knem: A generic and scalable kernelassisted intra-node mpi communication framework," Journal of Parallel and Distributed Computing, vol. 73, pp. 176-188, 2013.

[24] L. Chen, Y. Lu, Y. Yao, S. Peng, and L. Wu, "A well-balanced time warp system on multi-core environments," in Principles of Advanced and Distributed Simulation (PADS), 2011, pp. 1-9.

[25] R. Vitali, A. Pellegrini, and F. Quaglia, "Towards symmetric multithreaded optimistic simulation kernels," in Principles of Advanced and Distributed Simulation (PADS), 2012, pp. 211-220.

[26] J. Wang, K. Bahulkar, D. Ponomarev, and N. Abu-Ghazaleh, "Can pdes scale in environments with heterogeneous delays?" in Proc. of the 2013 ACM SIGSIM conference on Principles of advanced discrete simulation, 2013. 
Jingjing Wang is a PhD student in the Department of Computer Science at SUNY Binghamton. His research interests are in the areas of parallel discrete event simulation on multi-core systems.

Deepak Jagtap is a PhD student in the Department of Computer Science at SUNY Binghamton. His research interests are in the areas of parallel discrete event simulation on multi-core systems.

Nael Abu-Ghazaleh is an Associate Professor in the Department of Computer Science at SUNY Binghamton. His research areas are wireless and sensor networks, system security, and parallel discrete event simulation. He received his PhD from the University of Cincinnati in 1997.

Dmitry Ponomarev is an Associate Professor in the Department of Computer Science at SUNY Binghamton. His research areas are computer architecture, security, and parallel discrete event simulation. He received his PhD from SUNY Binghamton in 2003. 